Arbeitskampf betroffenen Arbeitnehmern fällt sowohl in das Risiko der Arbeitslosigkeit wie der Kurzarbeit. Nimmt man aber die Universalität und Gleichheit des sozialen Schutzes zum Ausgangspunkt, dann sind die Ausnahmen, die $\$$ I 16 Abs. 3 AFG normiert, im Hinblick auf Art. 3 GG wie auch der sozialstaatlichen Schutzpflicht zu legitimieren. Dabei dürfte wesentlich sein, daß das Kurzarbeitergeld im großen Maße das Wirtschafts- und Betriebsrisiko des Arbeitgebers auffängt und seine nach arbeitsrechtlichen Grundsätzen begründete Lohnzahlungspflicht über sozialversicherungsrechtliche Lösungen - mit erheblichen Lohneinbußen für die Arbeitnehmer - ablöst. ${ }^{30}$ Es widerspricht aber dem Gebot der Gleichbehandlung, wenn ein spezielles Betriebs- und Wirtschaftsrisiko, das Arbeitskampfrisiko, hiervon ausgenommen wird, ohne daß dies nachweisbar von anderen sachlichen Gesichtspunkten oder gar Konstruktionen wie der Parität im Arbeitskampf zwingend gefordert ist.

\title{
5. Zusammenfassung
}

Ohne hier eine eigene "Lösung « der verfassungsrechtlichen Kontroverse entwickeln zu können, bleibt als ein Fazit festzuhalten, daß plausible Ansätze wohl eher in den kollektiv strukturierten Rechtsgebieten der Koalitionsfreiheit und der Gleichheit im Sozialrecht zu suchen sein werden. Eine "Lösung " ganz anderer Art bringt aber das Verfahren selbst. Je länger die Entscheidung des Bundesverfassungsgerichts auf sich warten läßt, um so nachhaltiger müssen die Gewerkschaften auf Gedeih und Verderb die Praxis ihrer Tarifauseinandersetzungen unter dem neuen $\$$ II6 AFG einrichten. Und das mag dann das richterliche Vorverständnis beeinflussen, wonach alles so schlimm doch gar nicht gewesen sei, wenn man schon fast ein Jahrzehnt damit habe leben und zahlreiche Tarifauseinandersetzungen habe bestehen können. Auch an diesem Punkt zeigt sich wieder ein Strukturproblem des Arbeitsrechts, das wir vor allem vom Kündigungsschutzrecht her kennen. Da die Arbeitnehmer auf kollektive Gegenmacht und deren Anerkennung wie auch allgemein auf den Schutz durch das Recht angewiesen sind, hängen sie auch von der zügigen Durchsetzung dieses Rechts $\mathrm{ab}$, müssen sie sich doch sonst der Macht der Verhältnisse beugen.

\section{Rainer Wolf Hermann Heller*}

Am 5. November 1933 starb in Madrid ein Emigrant. Nach heute geltendem Recht wäre ihm das Asyl zu versagen gewesen, denn er kam aus Österreich und hatte sich vorher in England aufgehalten - zwei "sicheren Drittstaaten «. Ob entschieden worden wäre, er sei aufgrund des Verlusts seiner Frankfurter Professur politische Verfolgung bedroht gewesen, erscheint nach der Meßlatte des aktuellen Asylrechts so eindeutig nicht - immerhin hatte sich für seinen akademischen Mentor Karl Rad-

30 Vgl. E. Eichenhofer, Die rechtliche Behandlung von Kurzarbeit - vom privat- zum sozialrechtlichen Losungsansatz, RdA 1981, s. 208 ff.; K.-J. Bieback, in A. Gagel (Hg.), AFG, vor $\$ 63$, Rz. 131, 171 .

"Zugleich eine Besprechung von Hermann Heller, Gesammelte Schriften, 3 Bände, 2. Auf., hrsg. von Christoph Müller, Tübingen 1992 (J. C. B. Mohr), 298,- DM. Im folgenden wird aus den Arbeiten $\mathrm{Hel}$ lers unter Verweis auf Band und Seitenzahl dieser Ausgabe zitiert. 
bruch die »innerstaatliche Fluchtalternative« der »inneren Emigration « geboten -, und ob er, der 1925 aus der jüdischen Religionsgemeinschaft ausgetreten war, den Behörden die rassische Verfolgung hätte plausibilisieren können, ist gleichfalls nicht gewiß. Kurz: Die Karten für Hermann Heller dürften nach heutiger europäischer Asylrechtslage schlechter stehen als vor 60 Jahren, wenn er gezwungen wäre, von außen um Aufnahme in einem EG-Staat nachzusuchen.

Das Werk des Mannes aus Teschen an der Olsa genießt heute in Deutschland wohlwollendes Asyl - aber auch nur das. Das Wohlwollen verdankt es dem Begriff des "sozialen Rechtsstaates «, der geradezu synonym für sein akademisches Wirken gebraucht wird. Das ist eine sympathische, aber nicht ganz zutreffende Reduktion. Sie verweist auf eine angenehmere Wirkungsgeschichte als die bellizistischen Kurzformeln, durch die seine akademischen Antipoden mit Begriffen wie »totaler Staat» oder »innerstaatliche Feinderklärung " identifiziert werden. Jedoch hat Heller den Begriff des "sozialen Rechtsstaates « weder als erster verwandt ${ }^{1}$ noch fokussiert sich sein Werk auf eine Theorie des Sozialstaates. Mit gleichem Recht könnte man ihn als Theoretiker des »Kulturstaates « oder der Souveränität bezeichnen. Andererseits hat er zwar die Möglichkeit einer sozialen Transformation des Rechtsstaates als Alternative zum bürgerlichen Rechtsstaat der Besitzstandswahrung und zu Formen autoritärer Herrschaft herausgearbeitet, ihr aber nur ansatzweise spezifische rechtliche Form und Gestalt verliehen. Daß er vor allem staatstheoretische Interessen hatte, ist jedoch nur ein Grund dafür, daß seine juristische Rezeption in Deutschland keineswegs zum mainstream der Verfassungslehre führt. Viel hinderlicher erscheint, daß er den Begriff des »sozialen Rechtsstaates « mitunter auch mit dem Begriff des »sozialistischen Rechtsstaates " (II, 4 I 6) vertauscht hat.

Hellers wissenschaftliches Werk beginnt mit einer Kritik der nationalistischen Traditionslinie des Rechtshegelianismus. Mit »Hegel und der nationale Machtstaatsgedanke in Deutschland " (I, 2 I ff.) habilitierte er sich bei Gustav Radbruch in Kiel. Die politische Streitschrift "Sozialismus und Nation " $(\mathrm{I}, 437 \mathrm{ff}$.), die die damals relevanten politischen Ideenkreise zusammenfaßte, steht für sein Wirken in der politischen Bildung. Sein Aufsatz $»$ Die Krisis der Staatslehre« (II, 3 ff.) öffnet ihm 1926 den Zugang zum Kaiser-Wilhelm-Institut für ausländisches öffentliches Recht und Völkerrecht. Mit seinem Beitrag über den »Begriff des Gesetzes in der Reichsverfassung (II, $203 \mathrm{ff}$.) profiliert er sich 1927 vor der Vereinigung der Deutschen Staatsrechtslehrer als Kritiker Labands. Im gleichen Jahr veröffentlicht er seine staatstheoretische Pilotstudie "Die Souveränität« (II, 3 I ff.). In die aktuelle Politik interveniert Heller 1929 mit seinen Schriften "Europa und der Faschismus" (II, $463 \mathrm{ff}$.) und "Rechtsstaat oder Diktatur " (II, $443 \mathrm{ff}$.). Beachtung verdienen noch seine Arbeiten über »Grundrechte und Grundpflichten« (II, 28I) und »Politische Demokratie und soziale Homogenität « (II, $42 \mathrm{I})$. Sein Werk kulminiert in der »Staatslehre « (III, $77 \mathrm{ff}$.), die 1934 postum nach seinem Tod in der Emigration von Gerhart Niemeyer herausgegeben wurde.

Heller hat viele Bewunderer, aber wenig Schüler gefunden. Dies liegt nicht nur an dem frühen Tod, der den 42 jährigen ereilte. Seine Professur verdankte er der Intervention des Preußischen Kultusministers. Es ist symptomatisch, daß die ersten systematischen Bemühungen um eine Gesamtdarstellung seines Werkes nach I945 aus der Erwachsenenbildung ${ }^{2}$ und den Sozialwissenschaften ${ }^{3}$ stammen. Auch nach-

I Vgl. bereits Piloty-Schneider, Grundriß des Verwaltungsrechts, 1921

2 Klaus Meyer, Arbeiterbildung in der Volkshochschule, Stuttgart 1969; Hans Ràdle, Erwachsenenbildung und staatsburgerliche Erziehung, in: Paedagogica Historica 1969, S. $448 \mathrm{ff}$.

3 Wolfgang Schluchter, Entscheidung für den sozialen Rechtsstaat. Hermann Heller und die staatstheoretische Diskussion in der Weimarer Republik, 1968, 2. Aufl. Baden-Baden 1983. 
dem Martin Drath und Christoph Müller sein Werk 1971 in einer umfassenden Edition einer größeren Leserschaft wieder zugänglich gemacht hatten, änderte sich die Rezeptionslage nicht grundlegend. Die von kritischen Juristen ${ }^{4}$ eingeforderte Auseinandersetzung mit Heller blieb - auch in dieser Zeitschrift - zunächst weitgehend aus, weil zu Zeiten der Studentenbewegung die schrille Kritik der Kapitalexegetik an der "Sozialstaatsillusion ${ }^{5}$ die komplexen und keineswegs eindeutigen Begründungsansätze Heller übertönte. Die Rezeptionsansätze der achtziger Jahre ${ }^{6}$ blieben vorwiegend eine akademische Übung, weil inzwischen die politischen Rahmenbedingungen gewendet worden waren. Der soziale Rechtsstaat war durch die Deregulierungsdiskussion zur intellektuellen Demontage freigegeben, obwohl die Aktualität Hellers in mehreren Gedenkschriften ${ }^{7}$ mit guten Gründen belegt worden war. Andererseits konnte sich die neue ökologische Bewegung augenscheinlich nicht mit einem Werk befreunden, das der sozialen Gerechtigkeit mehr verpflichtet schien als dem Recht der Natur.

Heller wird ein Solitär bleiben. Er hat sich selbst nicht einer wissenschaftlichen Schule zugeordnet, und sein Werk wird auch nicht zu einer postumen Schulenbildung führen. Für eine exegetische Konservation ist es zu komplex. Es wirft auch in der "Staatslehre« mehr Forschungsperspektiven auf, als es Antworten gibt, sein Arbeitsspektrum als Leiter der Volkshochschule Leipzig und Bildungstheoretiker der Arbeiterbewegung, als politisch weitsichtiger Analytiker des italienischen Faschismus und hilfloser Helfer der vom Untergang bedrohten Weimarer Republik, als Professor für Staatsrecht an den Universitäten von Berlin und Frankfurt ist zu vielschichtig, sein politisches Bekenntnis als Kriegsfreiwilliger und Militärgerichtsakzessist, aktiver Kämpfer gegen den Kapp-Putsch, Kritiker des »Versailler Unrechtsvertrages (I, 639) und als Prozeßvertreter der abgesetzten sozialdemokratischen Regierung gegen den "Preußenschlag « ist zu facettenreich, sein intellektueller Kontext zu Hegel, Marx, Lasalle, den Austromarxisten, Max Weber, Karl Mannheim, Georg Jellinek und - horribile dictu - zu Hans Freyer und Carl Schmitt ist zu eigenständig, sein Changieren zwischen Sozial- und Rechtswissenschaften zu voraussetzungsvoll, seine methodologische Kritik an politisch ihm nahestehenden Wissenschaftlern wie Kelsen und Radbruch ist zu irritierend, um eine geschlossene Schar juristischer Jünger auf Dauer an sich zu binden. Wolfgang Abendroth ist ihm in bezug auf den sozialen Rechtsstaat gefolgt ${ }^{8}$, Peter Häberle hat die Theorie des Kul-

4 Vgl. dazu die Besprechung der Arbeit von Schluchter durch Jürgen Seifert, in: KJ 1968, 210.

5 Wolfgang Muller/Christel Neusüß, Die Sozialstaatsillusion und der Widerspruch von Lohnarbeit und Kapital, in: ProKla Sonderheft I (I971), S. 7 ff.

6 Vgl. dazu Joachim Blau, Sozialdemokratische Staatslehre in der Weimarer Republik. Darstellung und Untersuchung der staatstheoretischen Konzeptionen von Hermann Heller, Ernst Fraenkel und Otto Kirchheimer, Marburg 1980; Renate Granert, Die Staatsrechtslehre in der politischen Auseinandersetzung der Weimarer Republik, Freiburg 1980; Gerhard Robbers, Hermann Heller: Staat und Kultur, BadenBaden 1983; Stephan Albrecht, Hermann Hellers Staats- und Demokratieauffassung, Frankfurt 1983; Wolfgang Luthardt, Sozialdemokratische Verfassungstheorie in der Weimarer Republik, Opladen 1985; Thomas Vesting, Politische Einheitsbildung und technische Realisation. Über die Expansion der Technik und die Grenzen der Demokratie, Baden-Baden 1990; vgl. zu Leben und Werk Christoph Muller, Hermann Heller. Vom liberalen zum sozialen Rechtsstaat, in: KJ ( $\mathrm{Hg}$.), Streitbare Juristen. Eine andere Tradition, Baden-Baden 1988 , S. $268 \mathrm{ff}$

7 Vgl. dazu Christoph Müller/Ilse Staff, Der soziale Rechtsstaat. Gedächtnisschrift fur Hermann Heller. 1891-1933, Baden-Baden 1984; Wichtige Arbeiten daraus sind wiederabgedruckt in: Christoph Muller/Ilse Staff (Hg.), Staatslehre in der Weimarer Republik. Hermann Heller zu ehren. Frankfurt 1985 . Vgl. auch Wilfried Fiedler, Materieller Rechtsstaat und soziale Homogenitat. Zum so. Todestag von Hermann Heller, JZ 1984, $201 \mathrm{ff}$.

$8 \mathrm{Vgl}$. dazu Wolfgang Abendroth, Zum Begriff des demokratischen und sozialen Rechtsstaates im Grundgesetz der Bundesrepublik Deutschland, in: ders., Antagonistische Gesellschaft und politische Demokratie, Neuwied-Berlin 1967, S. $109 \mathrm{ff}$. 
turstaates ${ }^{9}$ und das Postulat der Offenheit der Verfassung $^{10}$ aufgegriffen, Friedrich Müller steht ihm wohl methodologisch am nächsten ${ }^{11}$. Sie tun dies nicht, um über ihren gemeinsamen Bezugspunkt ihre unterschiedlichen Positionen zu homogenisieren, sondern befestigen sie vielmehr gegeneinander. Auf Heller könnten sich viele Schulen berufen. Nicht weniger als Carl Schmitt darf Hermann Heller in Anspruch nehmen: complexio oppositorum ${ }^{12}$.

Hermann Heller gilt als der Staatsrechtslehrer der Sozialdemokratie. Er ist ein vehementer Kritiker der »anarchistischen Raserei unserer kapitalistischen Produktion « (II, 462), ein Protagonist der staatlichen Intervention und der »bewußten Ordnung der gesellschaftlichen Wirklichkeit nach einem einheitlichen Plane (III, 388), tritt für die möglichst weitgehende Beschränkung des Privateigentums, ein staatliches Außenhandelsmonopo] und die Sozialisierung der großen Unternehmen (II, 416) ein und scheut sich nicht, die Mittel des Klassenkampfes zu bejahen. Aber mit seinem Eintritt in die SPD gibt er zu Protokoll, er lehne den Historischen Materialismus und den proletarischen Internationalismus $a b^{13}$, statt dessen propagiert er die Vereinigten Staaten von Europa, betont unter machtpolitisch eingefärbter Affirmation die staatliche Souveränität, auch wenn sie sich »gegen das Recht absolut behauptet« (II, S. 185), tritt für einen »nationalen Kultursozialismus « (I, S. $437 \mathrm{ff}$.) ein und sieht in der nationalen Kulturgemeinschaft die Basis für die soziale Homogenität einer politischen Wertegemeinschaft. Im politischen Koordinatensystem der Weimarer Republik war Heller mit seinen ersten Positionen »in der deutschen Staatsrechtslehre der einsame Repräsentant der äußersten Linken ${ }^{\mathrm{T}}{ }^{4}$, mit seinen weiteren Aussagen stand er im Bereich des programmatisch nur zu häufig versagenden rechten Flügels der sozialdemokratischen Partei's. Auch damit verwirrt man potentielle Anhänger.

Heller setzt sich im staatstheoretischen Gefolge von Lasalle für die politische Reform der kapitalistischen Gesellschaft ein, und er steht als staatsrechtlicher Protagonist der Fundamentaldemokratisierung insoweit in der Tradition von Rousseau. Er definiert die Herausforderung des Staatswesens als Organisation "der Herrschaft des Volkes als Einheit über das Volk als Vielheit« (II, 99). Nur das »sich selbst bestimmende Volk kommt als "Träger der Gemeinschaftswerte« in Betracht (II, 374). Infolgedessen ist die Verfassung die »offene politische Form « (II, 377) eines durch inhaltliche Schranken nicht weiter limitierten demokratischen Prozesses. Daher bekämpft er den konstruierten Dualismus von formellem und materiellem Gesetz, mit dem der demokratische Prozeß an einer ganz entscheidenden Stelle amputiert und der monarchischen Exekutive die Organisationsgewalt über den Staatsapparat und den Staatshaushalt gerettet worden war. Gesetz ist für ihn die »von der Volkslegislative gesetzte Rechtsnorm « (II, 225) - tertium non datur. Die Verfassung gibt den Rahmen vor, der den Klassenkampf "in kulturermöglichende Formen bringt « (II, 374). Sie ist damit dann auch die Voraussetzung für eine »bewußte politische Einheitsbildung von unten nach oben« (II, 92, 425), und erst dadurch kann der Staat als »Organisation von Willensgegensätzen« (III, 265) zu einer »organisierten Entschei-

9 Vgl. dazu Peter Haberle, Kulturstaatlichkeıt und Kulturverfassungsrecht, 1982.

10 Peter Haberle, Die offene Geselischaft der Verfassungsinterpreten, in: ders., Verfassung als offentlicher Prozeß, Berlin 1978, S. $155 \mathrm{ff}$

i Friedrich Muller, Rechtsstaatliche Methodık und politische Rechtstheorıe, in: ders., Rechtsstaatliche Form - Demokratische Republik, Berlin 1977, S. 27 I ff.

12 Vgl. dazu Helmut Quaritsch (Hg.), Complexıo oppositorum. Über Carl Schmitt, Berlin 1988.

13 Vgl. dazu Schluchter, in: Muller/Staff (Hg.), Staatslehre (Fn. 6), S. 29.

is A bendroth, in: Muller/Staff (Fn. 12), S. 54 .

15 Die Mandarine der marxistuschen Lehre haben seine Staatslehre denn auch reichlich ungnadig aufgenommen; vgl. dazu die Rezension von Hans Mayer in der Zettschrift fur Sozıalforschung 1935, S. 277. 
dungs- und Wirkungseinheit« (III, $339 \mathrm{ff}$.) werden. Er ist ein Protagonist des "Verfassungspatriotismus."

Heller versucht, antagonistische Gegensätze durch eine Strategie des »Und « zu entpolarisieren: bürgerliche Freiheit und soziale Gleichheit, bürgerliches Kulturgut und proletarische Kultur, Nation und zwischenstaatliche Friedensordnung. Er will nicht die Ablösung des Rechtsstaates durch den Sozialstaat, sondern die Synthese: den sozialen Rechtsstaat. Sein Konzept des sozialen Rechtsstaates und des Kulturstaates zielt aber nicht zentral auf die politische und juristische Begründung öffentlicher Infrastruktur. Dies gelang Ernst Forsthoff mit seinen Analysen zur »Daseinsvorsorge« weit differenzierter. Der soziale Rechtsstaat ist für Heller untrennbar mit dem Begriff der sozialen Demokratie verbunden: „Die soziale Disparität kann aber summum jus zur summa injuria machen. Die radikalste formale Gleichheit wird ohne soziale Homogenität zur radikalsten Ungleichheit und die Formaldemokratie zur Diktatur der herrschenden Klasse« (II, 430). Heller betont die Funktionsbedingung der modernen Demokratie: Freiheit unter der Bedingung von Gleichheit (III, 207). Der soziale Rechtsstaat ist für Heller nicht nur eine sozialpolitische Option für den Weg aus Armut und Elend, sondern vor allem die demokratietheoretische Bedingung für die Etablierung des Parlamentarismus in der antagonistischen Gesellschaft. Er ist damit nicht lediglich eine mildtätige Erweiterung des Liberalismus, sondern das einzig wirksame politische Bollwerk gegen den Faschismus.

Heller betreibt die Synthese zwischen kritischer Realanalyse und normativen Entwürfen für eine bessere Zukunft. Deshalb changieren bei ihm Macht und Moral, Machtstaat und Kulturstaat, Klassenkampf und Wertegemeinschaft, nationale Souveränität und zwischenstaatliche Friedensordnung. Er ist ein Verteidiger der politischen Kompromisse und ein Theoretiker der Transformation scheinbar unvereinbarer Gegensätze. In seiner Staatslehre betont er die "Zukunftsschau « mit einer "gegenwartstranszendenten Orientierung " und fordert, die Wirklichkeit "auch unter dem Aspekt ihrer möglichen Veränderbarkeit" (III, I 52 ) zu betrachten. Er versucht um mit Musil zu sprechen - "Wirklichkeits-« und »Möglichkeitssinn« zu verbinden. Er analysiert daher nicht nur die Risiken und die Krisen, sondern er ventiliert auch die Chancen der Gesellschaft, die er schon 60 Jahre vor der »Erfindung des Politischen ${ }^{16}$ immer mehr auf Entscheidungen gegründet und damit als veränderbar ansah. Zumindest insoweit gehört er zur Moderne der Staatstheorie und ist durch den werkgeschichtlichen Alterungsprozeß auch nicht vom linken auf ihren rechten Flügel gewandert.

Ingeborg Maus hat die »ungebrochene Aktualität Hermann Hellers« darin gesehen, »daß alle wesentlichen in seinem Werk thematisierten Probleme... bis zur Stunde ungelöst « seien ${ }^{17}$. Nun darf man heute daran zweifeln, ob es nach wie vor »die weltgeschichtliche Bestimmung « der Arbeiterklasse sei, »in der Nation die sozialistische Idee zu verwirklichen « (I, 474). Damit entfallen zwei nicht nur für Heller wesentliche Fixpunkte: Die Zurückdrängung des Kapitalismus durch die staatliche Organisation der wirtschaftlichen Bedarfsdeckung kann nicht mehr als taugliches Mittel zur Bewältigung der Krisen der Marktwirtschaft gesehen und die Arbeiterbewegung kann nicht mehr als historisches Subjekt hypostasiert werden, das diesen Prozeß der Transformation als Projekt gesamtgesellschaftlicher Emanzipation durchsetzt. Unter diesem Verlust leidet heute jede kritische Theorie der Gesellschaft.

I6 Ulrich Beck, Die Erfindung des Politischen. Zu einer Theorie reflexiver Modernisierung. Frankfurt I993.

17 Maus, in: Múller/Staff (Fn. 12), S. 194. 
Heller ist ohne Zweifel eine adäquate staatsrechtliche Formulierung der Probleme der antagonistischen Gesellschaft der Weimarer Republik gelungen. Gilt dies auch für den Grad der aktuellen Differenzierung der postmodernen Gesellschaft? Antiquiert wirken ohne Zweifel die meisten seiner wirtschaftspolitischen Stellungnahmen. Thomas Vesting hat darüber hinaus gezeigt, daß Hellers staatstheoretische Grundannahmen auch durch die technische Realisation vor große Probleme gestellt werden $^{18}$. Christoph Müller hat das zentrale Problem für die Aktualität Hermann Hellers in der Verschiebung des politischen Zentralproblems von der sozialen auf die ökologische Frage gesehen ${ }^{19}$. Hier fällt es jedoch nicht schwer, mit dem Konzept Hellers von der staatsrechtlichen Entkoppelung antagonistischer gesellschaftlicher Probleme vom sozialen Rechtsstaat zum Postulat des ökologischen Rechtsstaates weiterzuschreiten. Der ökologische Rechtsstaat wäre danach der politische Prägestock für den Umbau der ökonomischen Nutzungsmuster natürlicher Ressourcen. So wie sich historisch eine soziale Marktwirtschaft nicht aus sich selbst heraus entfalten konnte, wohl aber die Polarisierung marktförmiger Vergesellschaftung durch den sozialen Rechtsstaat eingegrenzt werden konnte, wird die Vision einer ökologischen »Effizienzrevolution ${ }^{20}$, die wirtschaftliches Wachstum von der Produktion ökologischer Risiken abkoppeln und damit erst eine ökologische Marktwirtschaft schaffen könnte, nur durch die Schablone des ökologischen Rechtsstaates geformt werden können.

Allerdings wird dieser Begriff nur zu geringen Teilen mit genuin staatswissenschaftlichen Inhalten zu füllen sein. Ob die Entkoppelung des wirtschaftlichen Wachstums von der Produktion ökologischer Risiken gelingt, hängt nur peripher von der Entwicklung einer Theorie ökologischer Gerechtigkeit ab. ${ }^{21}$ Ökologische Problemdiagnose und zentrale Lösungsstrategien werden primär im Kontext naturwissenschaftlich-technischer Disziplinen entwickelt. Ihnen durch adäquate Instrumente zur Durchsetzung zu verhelfen, wird die Aufgabe des Umweltrechts sein, dem damit weniger materiell intervenierende als vielmehr moderierende, kataly tische, die endogenen ökologischen Potentiale von Wirtschaft und Technik freisetzende und diskursive Prozesse organisierende Funktionen zukommen. Damit ist jedoch eine andere Staatlichkeit verbunden, als sie Heller augenscheinlich vorschwebte. Hier besteht Modernisierungsbedarf für Hellers nur zu häufig etatistisch zugespitztes Politikverständnis, der föderalen ebenso wie dezentralen Organisationsformen des Staates mit Mißtrauen begegnete und dem Staat nur zu leicht die besseren Einsichts- und Handlungsformen zuschrieb.

Daß die technische Realisation die eigengesetzliche Basis des Staates und des Rechts untergräbt, haben Hellers Antipoden bereits frühzeitig mit stiller Trauer zur Kenntnis genommen ${ }^{22}$. Andererseits hat Heller die Dialektik von politischer Intervention und dezentraler Steuerung, die die moderne Staatsdiskussion beherrscht, durchaus anvisiert: "Je weiter der Rechtsstaat in die Arbeits- und Güterordnung eindringt, desto notwendiger wird die Beseitigung der staatseigenen zugunsten einer Selbstverwaltung (II, 458). Von daher dürfte die These, daß das Problem des modernen

18 Vesting (Fn. 5), S. $160 \mathrm{ff}$.

I9 Muller, Nachwort in Bd. 3 der Gesammelten Schriften, S. 450 ff.

$20 \mathrm{Vgl}$. dazu Ernst Ulrich von Weizsacker, Erdpolıtık. Okologische Realpolıtık an der Schwelle zum Jahrhundert der Umwelt. Darmstadt 1990, S. 142.

21 Vgl. dazu Rainer Wolf, Gerechugkett und Umweltschutz. Von subjektiv-rechtlichen Begrundungparadoxıen zu kollektıv-rechtlıchen Losungsansatzen. In: ARSP-Sonderheft 1994 (im Erscheinen).

22 Vgl. dazu Volker Neumann, Der harte Weg zum sanften Ziel. Ernst Forsthoffs Rechts- und Staatstheorie als Paradigma konservatıver Technikkrituk, in: Alexander Roßnagel (Hg.), Recht und Technık im Spannungsfeld der Kernenergiekontroverse, Opladen $198_{4}$, S. $88 \mathrm{ff}$. 
Staates nicht in der maximalen Direktionsgewalt über Güterproduktion und Verteilung, sondern in der optimalen Justierung der Koppelung zwischen Wirtschaft und Politik besteht und daß die Enthoheitlichung der Interventionsformen in die Wirtschaft nur zu einer stärkeren Politisierung der staatlichen Handlungsformen führt, Hellers Staats- und Rechtstheorie, die ja immer wieder betont, daß Recht und Staat die Gesellschaft nicht beliebig gestalten können (III, $366 \mathrm{ff}$.), so fern nicht sein.

Viel größere Bedrängnis droht Hellers Werk von seinen gesellschaftstheoretischen Grundannahmen. Die Arbeiterklasse ist als »natürlicher « Widerpart und »Totengräber« des Kapitalismus sozialstrukturell und als proletarisches Lebensmilieu soziokulturell erodiert. Die zyklischen Krisen des Kapitals reproduzieren sich nicht mehr auf der Grundlage des privaten Elends abhängig Beschäftigter, sondern vor dem Hintergrund eines im Vergleich zu den Lebzeiten Hellers erheblich gestiegenen sozialen Wohlstandes der ökonomischen Kerngruppen der Gesellschaft. Gleichwohl hat die marktförmige Vergesellschaftung ihre polarisierende Wirkung nicht verloren. Allerdings polarisiert sie aktuell viel weniger konfliktträchtig im Binnenverhältnis zwischen Lohnarbeit und Kapital als im Ausgrenzungsverhältnis zwischen der Erwerbsgesellschaft und denen, die von der Erwerbstätigkeit ausgeschlossen oder in ihr marginalisiert und diskriminiert sind: Arbeitslose, Alte, Alleinerziehende, Angehörige von sozialen und ethnischen Minderheiten, Ausländer, Armutsemigranten.

Statt eines fest formierten sozialen Milieus der Arbeiterklasse, das diese vorwiegend durch lebensgeschichtliche Ereignisse betroffenen Gruppen integrieren könnte, entfaltet sich ein nach tradiertem Rechts-Links-Schema schwerlich zu klassifizierendes, geschweige denn politisch zu vereinendes Patchwork marginalisierter Minderheiten. Durch Pluralisierung und Individualisierung sind sie im Prozeß der Mehrheitsdemokratie strukturell ohne Chance, mehrheitsfähig zu werden. Die daraus resultierende Organisationsschwäche der Klientelgruppen der Sozialpolitik bedingt deren aktuelle Artikulationsschwäche. Sie bleiben als Adressaten der Sozialpolitik ohne nachhaltige Aussicht, zu ihren eigenen politischen Akteuren werden zu können. Wo dies - wie in den ethnischen Ghettos der amerikanischen Großstädte - nicht zutrifft, heißt ihre militante Option nicht Klassen-, sondern Straßenkampf. Wenn an diesen sozialen Brennpunkten langfristig jeder Schnitt in das soziale Netz - und damit sind nicht nur die sozialen Transfers, sondern noch viel mehr die Wohnungs- und Stadtentwicklungspolitik gemeint - zu einer Umbuchung der eingesparten Mittel auf den Haushalt der Organe der inneren Sicherheit zur Folge hat, erhält auch Hermann Hellers Konzept der sozialen Homogenität als Basis des demokratischen Prozesses eine neue Aktualität.

Wo sich aber die modernen Formen sozialer Marginalisierung einmal verfestigt haben, gibt es für an Positionen der Emanzipation und Aufklärung orientierte Ansätze kaum Entwicklungschancen mehr, sie bestehen allenfalls darin, solche Polarisierungen zu vermeiden. Damit erhält das Programm sozialer Gerechtigkeit strukturkonservative Züge. Nicht mehr der grundlegende Wande] der in antagonistische Klassen gespaltenen Gesellschaft, sondern die Eindämmung der sozialen Disparitäten, asymmetrischen Zugangschancen zu Reichtum und Einfluß und selektiven Interessenberücksichtigungsmuster trägt die Theorie des Sozialstaates. Statt der offensiven Transformation der Marktwirtschaft in eine bessere Wirtschaftsverfassung wird die Dauerkompensation ihrer strukturellen Disparitäten und der durch konjunkturelle Krisenzyklen verschärften Kontraktionen ihrer sozialen Lebensqualität zur aktuellen Herausforderung für Sozialstaatskonzepte.

Diese werden dadurch nicht weniger krisenanfällig. Ihre Funktionsvoraussetzung ist das kontinuierliche Wachstum der sozialpolitischen Verteilungsmasse. Doch gerade diese Politik der monetären Transfers steht unter der Restriktion, daß ihr zentrales 
Ausgleichs- und Steuerungsmedium strukturell knapp geworden ist: Geld. Die fiskalische Stabilisierung des Sozialstaates hat eine prekäre Bedingung zur Voraussetzung: die Abschottung der Inseln relativen sozialen Wohlstands gegen den Rest der Welt. Dies mag gegen die Flut der Arbeits- und Armutsemigranten mit den Mitteln territorialer Exklusion noch gelingen, gegen die ökonomische Integration in die internationalen Finanzmärkte und die globale Arbeitsteilung, die viel entschiedener die nationale Basis der Systeme sozialer Sicherheit und die ökonomische Souveränität von Wohlfahrtsstaaten unterminieren, gibt es keine vergleichbaren hoheitlichen Mittel. Eine Alternative liegt zumindest theoretisch in der Besinnung darauf, daß Geld nur eine materielle Ressource der Gesellschaft ist. In einer Zeit, in der Geld fiskalisch kaum mehr disponibel ist, gleichwohl die Fähigkeiten, Kenntnisse und das Arbeitsvermögen vieler Menschen brachliegen, könnte die Mobilisierung dieser Ressourcen unerschlossene Potentiale zur Herstellung sozialer Gerechtigkeit eröffnen. ${ }^{23}$

Je problematischer die Finanzierung sozialer Gerechtigkeit wird, desto stärker wird der Druck auf nichtökonomische Integrationspotentiale. Und damit wird auch eine problematische Begrifflichkeit der Heller'schen Staatstheorie virulent. Wie kann kollektive Identität sich in einer sozialstrukturell entformierenden und in der Individualisierung privater Lebensentwürfe entsolidarisierenden Gesellschaft entstehen und ein »Wirbewußtsein und -gefüh $]_{\text {« }}\left(\mathrm{II},{ }_{428}\right.$ ) erzeugen? Die autoritäre Variante der Produktion gemeinschaftlicher Werte besteht in der Ausbeutung nationaler Stimmungen. Heller stellte dagegen, es sei der Glauben »an die Existenz einer gemeinsamen Diskussionsgrundlage ( $\mathrm{II}, 427)$. Doch er hatte kein blindes Vertrauen in die einheitsstiftende Kraft des Verfahrens. Entscheidend für ihn war, daß darüber die Hoffnung auf eine nachhaltige Änderbarkeit der sozialen Verhältnisse begründbar wird. Was geschieht, wenn die Chance der Veränderbarkeit keine Basis mehr findet?

\section{Werner Hill \\ Erinnerung an Ulrich Klug}

Es ist etwas schwierig, einen Nachruf auf einen Menschen zu schreiben, der so freundlich-lebendig, so verschmitzt und in einem guten Sinne listig war wie Ulrich Klug - er müßte doch eigentlich auch dem Tod noch ein Schnippchen geschlagen haben. Aber der Mann der Wissenschaft und der Politik, der Strafrechtler und Rechtsphilosoph, Staatssekretär und Justizsenator ist tot; seine Frau Ruth-Marion und seine Tochter Angelika Beate haben uns von ihrer Trauer um den »unerwartet im 80. Lebensjahr am 7. Mai 1993 Verstorbenen mitgeteilt. Viele Freunde und Weggenossen haben sich am I 7. Mai 1993 zur Trauerfeier in Köln-Rodenkirchen eingefunden und miterlebt, wie dem toten Ulrich Klug das Kunststück gelang, auf eine Weise beerdigt zu werden, die den Gefühlen von Christen und Juden, Atheisten und Agnostikern gleichermaßen entsprechen mochte: eine letzte, eine Abschiedslist des Vernunftmenschen Ulrich Klug und ein Vermächtnis, das Irrationale aller Weltanschauungen zu überwinden. »Eine humane menschliche Gesellschaft kann man sich

23 Vgl. dazu Claus Offe/Rolf G. Heınze, Organısıerte Eıgenarbett. Das Modell Kooperationsrıng, Frankfurt/M. 1990 . 